\title{
Reversible Formation of a Bose-Einstein Condensate
}

\author{
D.M. Stamper-Kurn, H.-J. Miesner, A.P. Chikkatur, S. Inouye, J. Stenger, and W. Ketterle \\ Department of Physics and Research Laboratory of Electronics, \\ Massachusetts Institute of Technology, Cambridge, MA 02139
}

(submitted)

\begin{abstract}
We present a method of adiabatically changing the local phase-space density of an ultracold gas, using a combination of magnetic and optical forces. Applying this method, we observe phase-space density increases in a gas of sodium atoms by as much as 50 -fold. The transition to Bose-Einstein condensation was crossed reversibly, attaining condensate fractions of up to $30 \%$. Measurements of the condensate fraction reveal its reduction due to interactions.
\end{abstract}

The physical properties of atomic gases change dramatically when quantum degeneracy is reached, i.e. when the ground state population approaches unity [1]. Recent successes in reaching quantum degeneracy with Bose gases [2 4] have relied on non-adiabatic, irreversible methods such as laser and evaporative cooling. The possibility of changing the ground state population by an adiabatic change in the trapping potential had been overlooked for quite some time [5]. Indeed, in the case of an ideal gas, adiabatic changes in the strength of the trapping potential do not change the ground state population [6,7]. However, Pinkse and collaborators [8] recently showed, both theoretically and experimentally, that by changing the form of the trapping potential, the population in the ground state can be changed adiabatically. For a non-degenerate gas the ground state population is identical to the phase-space density $\Gamma=n \lambda_{T}^{3}$, where $n$ is the density of the gas and $\lambda_{T}$ is the thermal de Broglie wavelength. Within the type of trap deformations considered in Ref. [8] the maximum increase of phase-space density is limited to a factor of 20 .

In this Letter, we show that a more general deformation of the trapping potential can increase the phasespace density by an arbitrary factor, and we describe an implementation of this scheme using a combination of magnetic and optical forces. Furthermore, we demonstrate the ability to cross the Bose-Einstein condensation (BEC) phase transition and to change the condensate fraction of a Bose gas in a reversible way.

Adiabatic increase in phase-space density. The type of trap deformations which we study can be understood with the following "two-box" model. Consider a classical gas of $N$ atoms confined in a box of volume $V_{0}=V_{1}+V_{2}$ with an initial phase-space density $\Gamma_{0}$. Suppose that the potential within a sub-volume $V_{2}$ of the box is lowered to a final well-depth $U$. In this final potential, the gas equilibrates at a temperature $T$, and the density in $V_{2}$ will be higher than that in $V_{1}$ by the Boltzmann factor $e^{U / k_{B} T}$. Using the condition of adiabaticity and constant particle number, one obtains the relative increase of phase-space density in $V_{2}$ compared to that in $V_{0}$ before compression:

$$
\ln \left(\Gamma_{2} / \Gamma_{0}\right)=\frac{U / k_{B} T}{1+\left(V_{2} / V_{1}\right) e^{U / k_{B} T}}
$$

For deep potential wells, where $U / k_{B} T \gg \ln \left(V_{1} / V_{2}\right)$, there is no increase in phase-space density since all of the gas becomes confined in $V_{2}$, and the adiabatic deformation corresponds simply to a uniform compression of the gas. For shallow potential wells $\left(U / k_{B} T \ll \ln \left(V_{1} / V_{2}\right)\right)$, the phase-space density in $V_{2}$ increases as $e^{U / k_{B} T}$. As $U$ is varied between these limits, the phase-space density increase reaches a maximum which is greater than $\left(V_{1} / V_{2}\right)^{1 / 2}$. Thus, by choosing an extreme ratio of volumes $V_{1} / V_{2}$, an arbitrarily large increase in phase-space density $\Gamma_{2} / \Gamma_{0}$ is possible.

To demonstrate this phase-space density increase in a gas of trapped atoms, a narrow potential well (analogous to $V_{2}$ ) was added to a broad harmonic potential (corresponding to $V_{1}$ ) by focusing a single infrared laserbeam at the center of a magnetic trap. First a gas of atomic sodium was evaporatively cooled to a temperature higher than the BEC phase transition temperture in the cloverleaf magnetic trap [9]. The number of atoms and their temperature were adjusted by varying the final radio frequency (rf) used in the rf-evaporative cooling stage [10]. Afterwards, the magnetically trapped cloud was decompressed by slowly reducing the currents in the magnetic trapping coils. Time-of-flight absorption imaging was used to characterize the cloud. The total number of atoms $N$ was determined by integrating the column density across the cloud, and $T$ was determined by one-dimensional Gaussian fits to the wings of the density distribution. From these, we determined the fugacity $z$ of the gas by the relation $g_{3}(z)=N\left(\hbar \bar{\omega} / k_{B} T\right)^{3}$, and then its phase-space density by $\Gamma_{0}=g_{3 / 2}(z)$, where $g_{n}(z)=\sum_{i=1}^{\infty} z^{i} / i^{n}$ [1]. Here, $\bar{\omega}$ is the geometric-mean trapping frequency of the magnetic trap, as determined by in situ measurements [12]. These phase-space density measurements were calibrated with images from magnetically trapped clouds at the phase transition.

The optical setup was similar to that used in Ref. [13]. The infrared laser power was gradually ramped-up from zero to a power $P_{c}$ at which the onset of BEC was seen in time-of-flight images of clouds released from the de- 
formed trap; this implied $\Gamma_{f}=g_{3 / 2}(1)=2.612$ for the final phase-space density. The depth of the optical potential well was given by $U_{c} / k_{B}=37 \mu \mathrm{K} P_{c} / w_{0}^{2}\left(\mu \mathrm{m}^{2} / \mathrm{mW}\right)$, where $w_{0}$ is the $1 / e^{2}$ beam-waist radius at the focus. The ramp-up time was made long enough to ensure that the trap deformation was adiabatic, but also short enough to minimize heating and trap loss. Ramp-up times of up to $10 \mathrm{~s}$ were used.
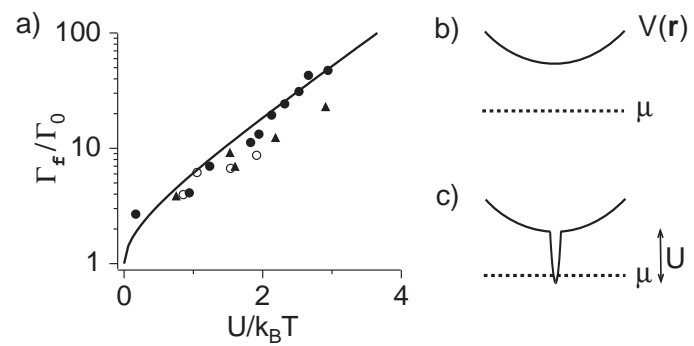

c)

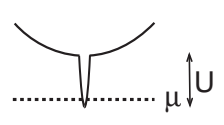

FIG. 1. Phase-space density increase to reach BEC vs. normalized well-depth (a). Various trap settings were used: $\bar{\omega}=2 \pi \times 100 \mathrm{~Hz}, w_{0}=9 \mu \mathrm{m}$ (triangles); $\bar{\omega}=2 \pi \times 100 \mathrm{~Hz}$, $w_{0}=18 \mu \mathrm{m}$ (open circles); and $\bar{\omega}=2 \pi \times 33 \mathrm{~Hz}, w_{0}=18 \mu \mathrm{m}$ (closed circles). The solid line gives the prediction of Eq. (2). (b) Prior to deformation, the harmonic trapping potential $V(r)$ holds a cloud above BEC the transition temperature $(\mu<0)$. (c) When a potential well with depth $U=-\mu$ is added, a small condensate forms.

Fig. 17a shows the increases in maximum phase-space density which were measured at three different settings of the trap parameters. A maximum increase by a factor of 50 was obtained. Condensates were observed in clouds with temperatures as high as $5 \mu \mathrm{K}$. Further increases were hindered by limitations in laser power and by limits to the ramp-up time set by the various heating and loss processes in the deformed trap. The scatter in the data is primarily due to statistical errors in our measurements of $U_{c} / k_{B} T$, at the level of $30 \%$, which arise from measurements of $P_{c}$ and $w_{0}$, and in the determination of the transition point.

The well-depth $U_{c}$ required to reach BEC can be understood by a simple model depicted in Fig. 1. We begin with a gas in a harmonic trap above the BEC transition temperature, i.e. its chemical potential $\mu<0$ (Fig. 1 $1 \mathrm{~b}$ ). Its initial phase-space density is given by $\Gamma_{0}=g_{3 / 2}(z)$, where $z=e^{\mu / k_{B} T}$ is the fugacity. In Fig. 迎, a narrow potential with depth $U_{c}$ is added until $U_{c}=-\mu$, at which point the gas begins to Bose condense. The phase-space density thus reaches the critical value $g_{3 / 2}(1)$, so that the increase in maximum phase-space density is given by

$$
\frac{\Gamma_{f}}{\Gamma_{0}}=\frac{g_{3 / 2}(1)}{g_{3 / 2}\left(\exp \left(-U_{c} / k_{B} T\right)\right)} .
$$

This prediction, shown in Fig. 1a, describes our data well, and accounts for the universal behaviour of our measurements over a wide range of temperatures and well-depths.

Note that in this simple model we made the implicit assumption that the initial and final temperatures of the cloud were equal. We can remove this assumption by considering instead the condition of constant entropy. The entropy per non-condensed particle in a harmonically confined Bose gas is determined uniquely by its fugacity $z$ [8]:

$$
\frac{S}{N}(z)=4 \frac{g_{4}(z)}{g_{3}(z)}-\ln z .
$$

This equation describes the entropy of the gas before compression, with the fugacity given by $z_{0}$. After compression, because of the small volume of the potential well, the entropy per particle is approximately that of a harmonically trapped gas (Eq. (3)) with fugacity $z_{f}=e^{-U_{c} / k_{B} T}$. Here $T$ is the final temperature of the gas, which is generally higher than the initial temperature [15]. Constant entropy then implies $z_{0}=z_{f}$, and thus one obtains Eq. (2) where $T$ is now the final temperature, as we have plotted in Fig. 1 1 a.

One may also consider the process of adiabatically increasing the phase-space density as a change in the density of states $D(\epsilon)$ of the system. By increasing the welldepth in a small region of the trap, we lowered only the energy of the ground state and a few excited states. Thus $\Gamma$, a local quantity, is maximally increased as the ground state energy is brought ever closer to the chemical potential, while the entropy, a global property of the gas, is unchanged by the minimal modification of $D(\epsilon)$.

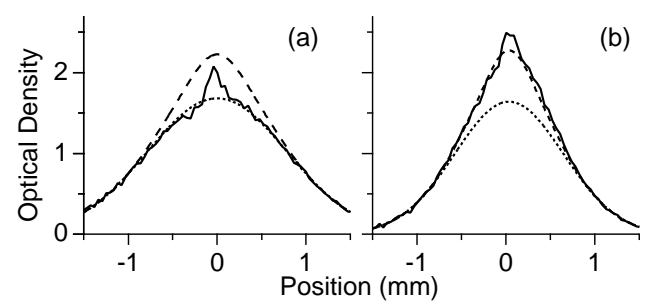

FIG. 2. Momentum distributions of the thermal cloud at the BEC phase transition show no Bose-enhancement for the deformed trap (a), but a clear Bose-enhancement for the purely magnetic trap (b). Both distributions show a small condensate peak. Lines show a Maxwell-Boltzmann distribution (dotted) and a Bose-Einstein distribution (for $z=1$ ) (dashed) for clouds in a harmonic trap, which were fit to the thermal wings. The momentum distribution is shown as a profile across absorption images taken after $40 \mathrm{~ms}$ of ballistic expansion.

The fact that global properties of the gas are not affected by the trap deformation can also be seen in the momentum distributions probed by time-of-flight imaging 9.16$]$. The onset of BEC in the combined optical and magnetic trap is signaled only by the formation of a condensate peak. The remaining thermal cloud is well fit by a Maxwell-Boltzmann distribution, which describes a magnetically trapped cloud far from condensation (Fig. 2a). In contrast, at the BEC transition in the 
harmonic magnetic trap, the momentum distribution of the thermal cloud is clearly Bose-enhanced at low momenta (Fig. 2b).

Adiabatic condensation. We now turn to the studies of adiabatic, i.e., reversible, condensate formation. A cloud of about $50 \times 10^{6}$ atoms was evaporatively cooled down to the the transition temperature in the magnetic trap, at trap frequencies of $\omega_{r}=2 \pi \times 20 \mathrm{~Hz}$ and $\omega_{z}=2 \pi \times 13 \mathrm{~Hz}$ in the radial and axial direction, respectively. The power of the infrared laser beam (of radius $\left.w_{0}=20 \mu \mathrm{m}\right)$, was ramped up over $1 \mathrm{~s}$ and held at a constant power for a dwell time of $1.5 \mathrm{~s}$. Condensate fractions as small as $1 \%$ could be distinguished from the normal fraction by their anisotropic expansion in timeof-flight images [2, 3]. The condensate number $N_{0}$ was determined by subtracting out the thermal cloud background using Gaussian fits to the thermal cloud in regions where the condensate was clearly absent.

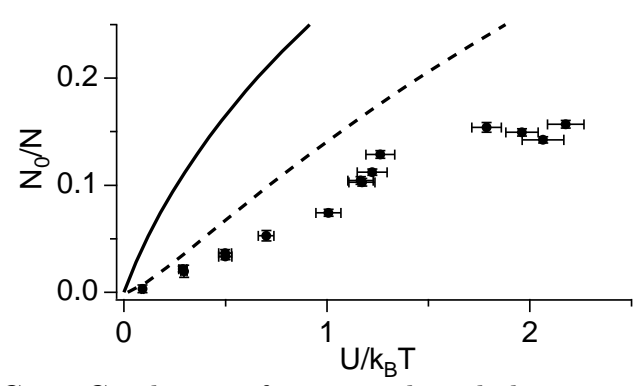

FIG. 3. Condensate formation by adiabatic trap deformation. The condensate fraction is plotted against the (normalized) well-depth. Lines show predictions for an ideal gas (solid) and for an interacting gas (dashed).

As shown in Fig. 3, the adiabatic trap deformation yielded condensate fractions of up to $15 \%$; we observed condensate fractions of $30 \%$ with different trap settings. By varying the dwell time, we confirmed that clouds for which $U / k_{B} T<1.5$ suffered no significant losses of condensate number due to three-body decay or heating, whereas those points with higher values of $U / k_{B} T$ were affected by such losses.

For an ideal Bose gas, the result of this adiabatic change can be understood as follows. Before compression, the cloud of $N$ particles at the BEC transition has an entropy $S_{i}$ given by Eq. (3) as $S_{i}=N \times 4 g_{4}(1) / g_{3}(1)$. After compression, the situation is similar to that indicated in Fig. 1c, i.e. because of the small volume of the attractive well, the cloud is well-described as a harmonically trapped gas with $\mu=-U$. Thus, accounting for the fact that condensate particles carry no entropy and again using Eq. (3), which gives the entropy per non-condensed particle, we equate the entropy before and after compression and obtain

$$
\frac{N_{0}}{N}=1-\frac{4 g_{4}(1) / g_{3}(1)}{4 g_{4}\left(e^{-U / k_{B} T}\right) / g_{3}\left(e^{-U / k_{B} T}\right)+U / k_{B} T} .
$$

However, this simple prediction does not describe our findings well. The theory described above has two shortcomings. First, the approximation of using Eq. (3) for the deformed trap is not strictly valid. However, calculations which accounted for the true shape of the deformed potential changed the prediction of Eq. (4) only for $U / k_{B} T>1$, and only slightly improved the fit to our data.

A second shortcoming is the neglect of interactions. It has been shown that in harmonic potentials, the condensate fraction in a Bose gas with repulsive interactions is reduced in comparison to that of an ideal gas [17, 18]. To estimate this effect in our system, we use the "semi-ideal" model of Ref. [18. The thermal cloud is described as an ideal gas for which the chemical potential is raised by $g n_{0}=4 \pi \hbar^{2} a n_{0} / m$, where $n_{0}$ is the maximum condensate density, $m$ the mass of sodium, and $a=2.75 \mathrm{~nm}$ its scattering length [19]. This simply corresponds to using Eq. (4) with the substitution $U \rightarrow U-g n_{0}$. We determined $n_{0}$ using the Gross-Pitaevskii equation in the Thomas-Fermi limit [20], and a harmonic approximation for the deformed trap potential at its center.

This approach predicts a significant reduction of the condensate fraction (Fig. 3, dashed line), and the improved agreement with our data is strong evidence for this effect. In contrast to related studies in purely harmonic traps 9, 16], which did not show evidence for interaction effects, this depletion is strongly enhanced by the shape of the potential we are using. The mean-field energy of the condensate $g n_{0}$ is large because the condensate forms in the tight optical potential, while the transition temperature $T_{c}$ is small since it is determined by the weak magnetic potential.

The reversibility of crossing the BEC phase transition was demonstrated by preparing a magnetically trapped cloud just above $T_{c}$. We then sinusoidally modulated the power of the infrared light at $1 \mathrm{~Hz}$, between zero and $7 \mathrm{~mW}$. This modulation frequency was significantly smaller than the magnetic trap frequencies $\left(\omega_{r}=2 \pi \times\right.$ $48 \mathrm{~Hz}$ and $\left.\omega_{z}=2 \pi \times 16 \mathrm{~Hz}\right)$. These low frequencies and a large optical focus $\left(w_{0}=18 \mu \mathrm{m}\right)$ were used to minimize trap loss due to inelastic collisions.

During the first seven condensation cycles the condensate fraction oscillated between zero and 6\% (Fig. 4); later probing showed repeated condensation for at least 15 cycles. The peak of these oscillations decreased slowly in time. The temperature also oscillated, with an amplitude of about $100 \mathrm{nK}$, while gradually rising by about $10 \mathrm{nK} / \mathrm{s}$. This heating and the decrease of the peak condensate fraction are consistent with similar behaviour in clouds held at a constant infrared power, which result from beam jitter, spontaneous scattering, and three-body decay [13]. Thus, within the stability limitations of our optical setup, the repeated crossing of the BEC phase transition appears fully adiabatic. 


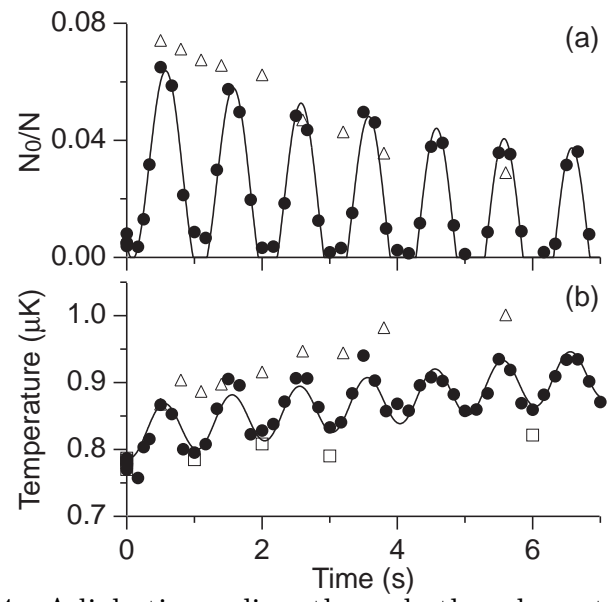

FIG. 4. Adiabatic cycling through the phase transition. Shown is the condensate fraction (a) and the temperature (b) vs time for the case of a modulated infrared beam (closed circles), an infrared beam ramped up to a constant power (open triangles), and no infrared light (open squares). The solid lines are guides to the eye.

This method of creating condensates provides insight into their formation, which was recently studied experimentally [21] and theoretically [22]. For example, in the experiment described above, the condensate fraction was found to lag about $70 \mathrm{~ms}$ behind the modulation of the laser power, which is a measure for the formation time.

In other experiments, by switching on the infrared light instantly, we observed condensation on timescales much faster than the oscillation periods in the magnetic trap and along the weakly confining axis of the optical trap. The resulting condensates showed striations in time-offlight images, indicating that the condensates formed into excited states of the deformed potential. Such studies of shock-condensation might give new insight into the formation of quasi-condensates and condensation into excited states 23,24.

In conclusion we have demonstrated the adiabatic Bose-Einstein condensation of an ultracold gas of atomic sodium. Changes in the trapping potential resulted in large phase-space density increases and allowed for repeated crossings of the BEC phase transition. This method allows for detailed studies of condensate formation and the phase transition. The combined trapping potential widens the range of trap parameters over which BEC can be studied. This was used to strikingly enhance the role of interactions, and led to higher transition temperatures (up to $5 \mu \mathrm{K}$ ) than achieved in purely magnetic traps.

We thank Michael R. Andrews for discussions. This work was supported by the Office of Naval Research, NSF, Joint Services Electronics Program (ARO), NASA, and the David and Lucile Packard Foundation. A. P. C. acknowledges additional support from the NSF, D. M. S. -K. from JSEP, and J. S. from the Alexander von Humboldt-Foundation.
[1] A. Griffin, D.W. Snoke, and S. Stringari, Bose-Einstein Condensation (Cambridge University Press, Cambridge, 1995); Georgia Southern University BEC home page, http://amo.phy.gasou.edu/bec.htm

[2] M.H. Anderson et al., Science 269, 198 (1995).

[3] K.B. Davis et al., Phys. Rev. Lett. 75, 3969 (1995).

[4] C.C. Bradley, C.A. Sackett, and R.G. Hulet, Phys. Rev. Lett. 78, 985 (1997), see also: C.C.Bradley et al., Phys. Rev. Lett. 75, 1687 (1995).

[5] W. Ketterle and D.E. Pritchard, Phys. Rev. A 46, 4051 (1992).

[6] F. Reif, Fundamentals of Statistical and Thermal Physics (McGraw-Hill Book Company, New York, 1965)

[7] For weakly interacting Bose gases this argument has to be modified; see: M. Houbiers, H.T.C Stoof, and E.A. Cornell, Phys. Rev. A 56, 2041 (1997).

[8] P. W. H. Pinkse et al., Phys. Rev. Lett. 78, 990 (1997).

[9] M.-O. Mewes et al., Phys. Rev. Lett. 77, 416 (1996).

[10] W. Ketterle and N.J. van Druten, in Advances in Atomic, Molecular, and Optical Physics, edited by B. Bederson and H. Walther (Academic Press, San Diego, 1996), Vol. 37, p 181.

[11] This assumes an ideal Bose gas and harmonic confinement; see: V. Bagnato, D. E. Pritchard, and D. Kleppner, Phys. Rev. A 35, 4354 (1987).

[12] D. M. Stamper-Kurn et al., preprint cond-mat/9801262 (unpublished).

[13] D. M. Stamper-Kurn et al., Phys. Rev. Lett. 80, 2027 (1998).

[14] K. Huang, Statistical Mechanics, second edition (Wiley, New York, 1987).

[15] This approximation is valid for shallow potential wells $\left(U / k_{B} T \ll \ln \left(V_{1} / V_{2}\right)\right)$, as discussed in the two-box model. Numerical calculations confirmed its validity for all data in Fig. 1a. Note that the effective volume of the magnetic trap $\left(V_{1}\right)$ increases with temperature.

[16] J. R. Ensher et al., Phys. Rev. Lett. 77, 4984 (1996).

[17] V.V. Goldman, I.F. Silvera, and A.J. Leggett, Phys. Rev. B 24,2870 (1981); R.J. Dodd, K. Burnett, M. Edwards, and C.W. Clark, Acta Physica Polonica 93,45 (1998); S. Giorgini, L.P. Pitaevskii and S. Stringari, Phys. Rev. A 54, R4633 (1996).

[18] M. Naraschewski and D.M. Stamper-Kurn, preprint cond-mat/9803216 (unpublished).

[19] E. Tiesinga et al., J. Res. Natl. Inst. Stand. Technol. 101, 505 (1996).

[20] G. Baym and C.J. Pethick, Phys. Rev. Lett 76, 6 (1996).

[21] H.-J. Miesner et al., Science 279, 1005 (1998).

[22] C.W. Gardiner, P. Zoller, R.J. Ballagh, and M.J. Davis, Phys. Rev. Lett. 79, 1793 (1997).

[23] Yu. Kagan, B.V. Svistunov, and G.V. Shlyapnkov, Sov. Phys. JETP 75, 387 (1992).

[24] C.W. Gardiner et al., preprint cond-mat/9801027 (unpublished). 